Gut, 1986, 27, S1, 111-115

\title{
Use of branched chain amino acids for treating hepatic encephalopathy: clinical experiences
}

\author{
F ROSSI FANELLI, C CANGIANO, L CAPOCACCIA, A CASCINO, F CECI, \\ M MUSCARITOLI, AND G GIUNCHI \\ From the Third Department of Internal Medicine, University of Rome "La Sapienza, " Rome, Italy
}

SUMMARY The efficacy of branched chain amino acids in two consecutive clinical studies in patients with severe hepatic encephalopathy was tested. In the preliminary uncontrolled study 19 patients with grade 3-4 hepatic encephalopathy were given an intravenous solution containing leucine $11 \mathrm{~g} / \mathrm{l}$, isoleucine $9 \mathrm{~g} / \mathrm{l}$, and valine $8.4 \mathrm{~g} / \mathrm{l}$ in $20 \%$ dextrose. A complete recovery of mental state was obtained in all patients in a mean time of 20.5 hours. In a subsequent controlled study 40 patients with grade 3-4 hepatic encephalopathy were randomly assigned to receive intravenous branched chain amino acid in $20 \%$ dextrose (group A) or oral lactulose (group B). Twelve patients $(70.6 \%)$ in group A and eight $(47 \%)$ in group B regained consciousness in a mean time of 27.6 and 31.5 hours, respectively. The difference in the recovery rate between the two groups, although evident, was not significant. Intravenous branched chain amino acids are thus at least as effective as lactulose in reversing hepatic coma.

These data argue strongly in favour of a therapeutic effect of branched chain amino acids in the treatment of hepatic encephalopathy in patients with chronic liver failure.

The characteristic plasma amino acid pattern, with high aromatic and low branched chain amino acids observed in chronic liver failure, is thought to have a causal role in the pathogenesis of hepatic encephalopathy. Brain accumulation of aromatic amino acids may induce a profound change in neurotransmitters' synthesis leading to hepatic encephalopathy. The administration of branched chain amino acids may improve hepatic encephalopathy either by reducing aromatic amino acid outflow from muscle proteins or by inhibiting their brain uptake through direct competition for a common carrier.

In chronic liver failure the severe catabolic state and the consequent degradation of body protein are the major determinants of the characteristic amino acid imbalance with increased aromatic amino acid, phenylalanine, tyrosine, tryptophan, and decreased branched chain amino acid, valine, leucine, isoleucine values (Fig. 1). ${ }^{1}$

As aromatic amino acid and branched chain amino acid are transported into the brain through a common carrier system in the blood brain barrier, ${ }^{2}$ brain uptake of aromatic amino acid may be favoured by the reduction in plasma branched chain

Correspondence to: Dr F Rossi Fanelli. Laboratory of Clinical Nutrition. University of Rome, Clinica Medica 3. Viale Dell Universita 37. (0)185 Rome. Italy. amino acid values. According to the hypothesis advanced by Fischer and Baldessarini in 1971, ${ }^{3}$ brain accumulation of aromatic amino acid may lead to a change in the synthesis of neurotransmitters, resulting in a depletion of putative neurotransmitters such as norepinephrine and dopamine, and an increased synthesis of false or weak neurotransmitters such as octopamine and phenylethanolamine and the inhibi-

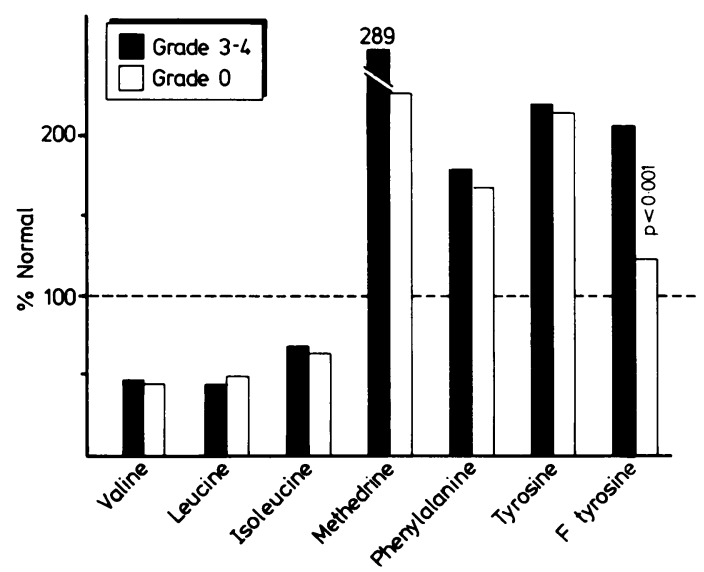

Fig. 1 Plasma amino acid pattern in chronic liver failure. 
tory neurotransmitter serotonin. Such a disturbance in neurotransmitter synthesis is thought to be responsible for the neurological disorders and coma seen in patients with chronic liver failure.

The part played by aromatic amino acid in the pathogenesis of hepatic encephalopathy has recently been confirmed by the finding that in normal dogs brain perfusion through the carotid arteries with a solution containing phenylalanine and tryptophan was followed within six hours by a reversible, hepatic like coma, ${ }^{4}$ accompanied by a clinically important rise in cerebrospinal fluid concentrations of phenylalanine, tryptophan, tyrosine, octopamine and phenylethanalamine (Fig. 2). In a later series of

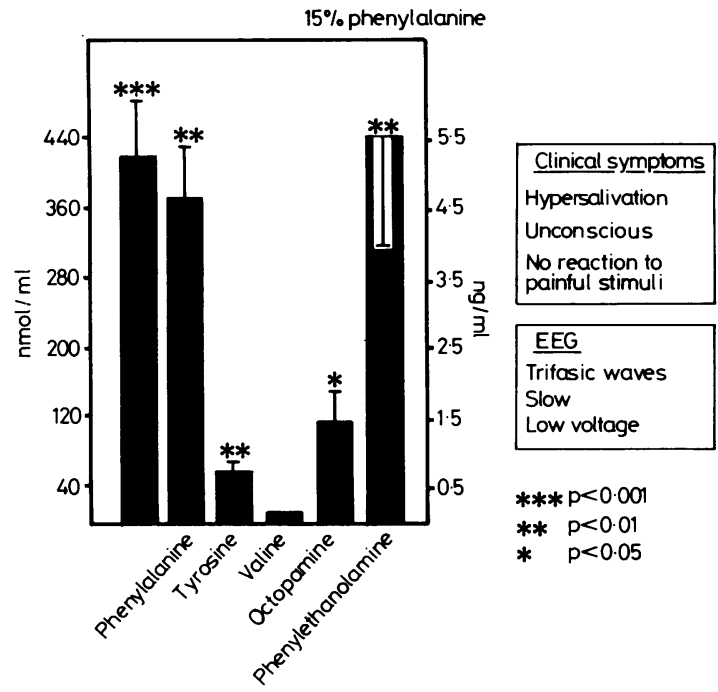

Fig. 2 Clinical, electroencephalografic, and cerebrospinal fluid biochemical modifications during intracarotid infusion of phenylalanine and tyrosine in normal dogs. experiments, using the same experimental model, it was shown that hepatic like coma induced by phenylalanine and tryptophan could be totally prevented by simultaneous infusion of the three branched chain amino acids. ${ }^{4}$ As a possible consequence of their characteristic regulatory influence on muscular protein turnover, ${ }^{5-7}$ branched chain amino acids have been shown to be particularly effective in reducing plasma concentrations of aromatic amino acid in chronic liver failure ${ }^{8}$ (Table 1). Branched chain amino acids should therefore be effective in the treatment of hepatic encephalopathy. They are thought to be able to interrupt the chain of events linking the hypercatabolic state to hepatic encephalopathy in at least three possible ways (Fig. 3): (i) They reduce protein breakdown and stimulate protein synthesis, (ii) they normalise the plasma amino acid pattern either by decreasing proteolysis or by increasing the use of aromatic amino acid for protein synthesis, (iii) they provide the bulk of competition across the blood brain barrier, thus preventing aromatic amino acid from accumulating in the brain.

\section{Clinical studies}

To test the clinical efficacy of branched chain amino acid in the treatment of hepatic encephalopathy two consecutive studies were carried out in patients with cirrhosis and severe encephalopathy (grade 3-4). ${ }^{9-10}$ In the preliminary uncontrolled study ${ }^{9} 19$ patients with grade 3-4 hepatic encephalopathy received an intravenous solution containing branched chain amino acid alone (leucine $11 \mathrm{~g} / \mathrm{l}$, isoleucine $9 \mathrm{~g} / \mathrm{l}$, valine $8.4 \mathrm{~g} / \mathrm{l}$ ) in $20 \%$ dextrose. The solution was delivered at a constant rate of $60 \mathrm{ml} / \mathrm{hour}$ for 24 hours, thereafter at $80 \mathrm{ml} /$ hour by means of a peristaltic pump. Precipitating events received appropriate treatment, but no specific treatment for

Table 1 Variations (mean(SE)) of plasma amino acid concentrations during glucose (solution A) or glucose + branched chain amino acid (solution B) infusion.

\begin{tabular}{|c|c|c|c|c|c|c|c|}
\hline & Preinfusion & One hour & Three hours & Six hours & 12 hours & 24 hours & Postinfusion \\
\hline \multicolumn{8}{|l|}{ Phenylalanine } \\
\hline Solution A & $1 \cdot 26(0 \cdot 16)$ & $1 \cdot 01(0 \cdot 16)$ & $1.01(0 \cdot 13)$ & $0.95(0 \cdot 11)$ & $1 \cdot 01(0 \cdot 13)$ & $0.91(0 \cdot 08)$ & $1.28(0 \cdot 15)$ \\
\hline Solution B & $1.54(0.32)$ & $1 \cdot 13(0 \cdot 25)$ & $0.51(0 \cdot 15)$ & $0.58(0 \cdot 10)$ & $0.45(0.07)$ & $0 \cdot 65(0 \cdot 05)$ & $1.45(0.20)$ \\
\hline \multicolumn{8}{|c|}{ Total tryptophan } \\
\hline Solution A & $0.90(0 \cdot 20)$ & $0.77(0 \cdot 12)$ & $0.92(0 \cdot 16)$ & $0.73(0.07)$ & $0.82(0.17)$ & $0 \cdot 71(0 \cdot 11)$ & $0.87(0.08)$ \\
\hline Solution B & $1 \cdot 01(0 \cdot 21)$ & $0 \cdot 82(0 \cdot 13)$ & $0 \cdot 73(0 \cdot 13)$ & $0.50(0 \cdot 13)$ & $0.52(0.09)$ & $0 \cdot 46(0 \cdot 10)$ & $0.81(0.23)$ \\
\hline \multicolumn{8}{|c|}{ Free tryptophan } \\
\hline Solution A & $0 \cdot 14(0 \cdot 01)$ & $0 \cdot 12(0 \cdot(02)$ & $0.08(0 \cdot 01)$ & $0.09(0 \cdot 02)$ & $0.09(0.01)$ & $0.09(0.01)$ & $0.09(0.02)$ \\
\hline Solution B & $0 \cdot 14(0 \cdot 01)$ & $0 \cdot 11(0 \cdot 01)$ & $0 \cdot 08(0 \cdot 01)$ & $0.05(0 \cdot 01)$ & $0.04(0.01)$ & $0 \cdot 04(0 \cdot 01)$ & $0 \cdot 15(0 \cdot 01)$ \\
\hline \multicolumn{8}{|l|}{ Tyrosine } \\
\hline Solution A & $2 \cdot 34(0 \cdot 24)$ & $1.87(0.17)$ & $1.79(0 \cdot 16)$ & $1 \cdot 68(0 \cdot 16)$ & $1.78(0.20)$ & $1.65(0 \cdot 23)$ & $2 \cdot 07(0 \cdot 17)$ \\
\hline Solution B & $2.37(0 \cdot 15)$ & $2.07(0.19)$ & $1.47(0.24)$ & $1 \cdot 20(0 \cdot 21)$ & $1.00(0.14)$ & $0 \cdot 80(0 \cdot 06)$ & $2 \cdot 11(0 \cdot 17)$ \\
\hline
\end{tabular}




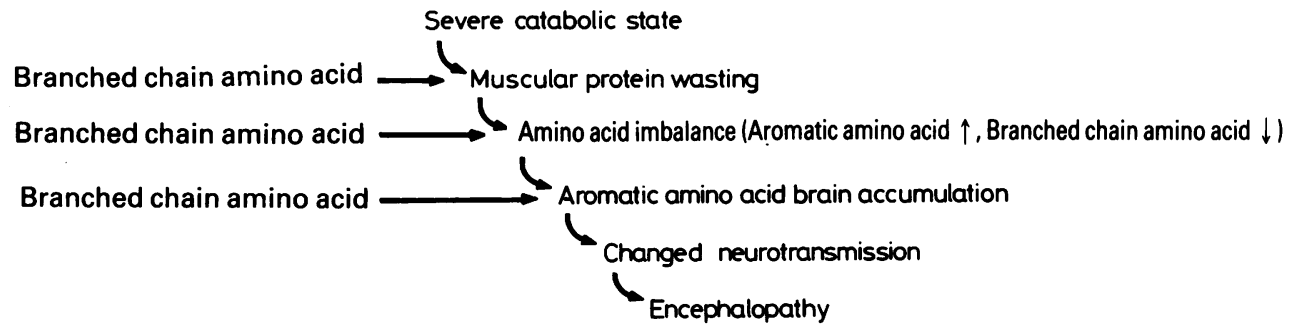

Fig. 3 Possible interference of branched chain amino acid on chain of events leading to hepatic encephalopathy.

hepatic encephalopathy was given. Complete recovery of the mental state - that is, grade 0 hepatic encephalopathy - was obtained in all patients two and a half to 48 hours (mean $20 \cdot 5$ (SE) 3.75). The time of arousal was closely related to the severity of the events that had precipitated encephalopathy (Fig. 4).

Encouraged by the favourable results of this preliminary study, a prospective controlled study was undertaken, in which the efficacy of the branched chain amino acid in $20 \%$ dextrose was compared with that of lactulose. ${ }^{10}$ Early criteria included the presence of liver cirrhosis, grade 3-4 hepatic encephalopathy, and the absence of hepatorenal syndrome.

Forty patients were randomly allocated to receive either branched chain amino acid or lactulose. Both groups were given intravenous glucose in isocaloric amounts. Precipitating events received appropriate treatment. Based on the results of the previous study, ${ }^{9}$ a time limit of 48 hours was fixed to assess

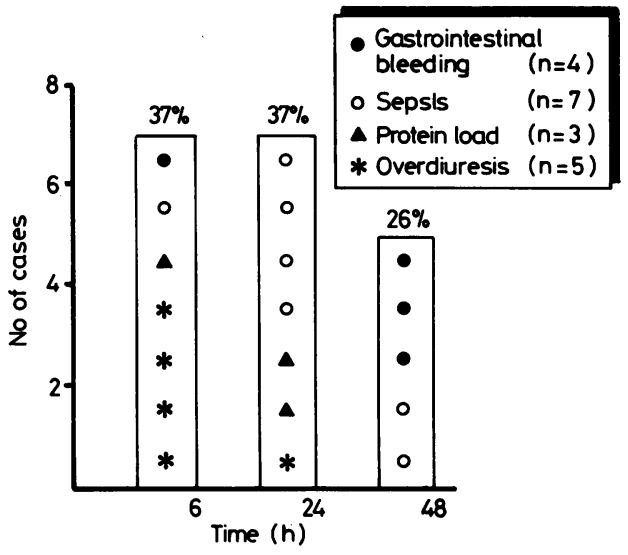

Fig. 4 Time of arousal related to precipitating factors observed in 19 patients with grade 3-4 hepatic encephalopathy receiving intravenous branched chain amino acids. the efficacy of the treatment. If mental recovery occurred within 48 hours treatment was continued for a further 48 hours and then discontinued. Thereafter, patients in both groups received a protein restricted diet plus lactulose in sufficient amounts to ensure a cathartic effect. If mental recovery did not occur within 48 hours the two treatments were combined. Mental recovery was considered to have occurred when the patients regained consciousness - that is, grade 0 hepatic encephalopathy, according to the classification of Adams and Foley. ${ }^{11}$

Three patients in each group were lost from the study, the data therefore refer to 34 patients. The two groups were comparable as far as sex, type of cirrhosis, precipitating events and liver function tests were concerned.

Table 2 shows the clinical results. Twelve of the 17 patients $(70.6 \%)$ receiving branched chain amino acid regained consciousness in a mean time of 27.6 $(6 \cdot 48)$ hours. In the group receiving lactulose eight of the seventeen patients ( $47 \%$ ) were fully alert in a mean time of $31.5(4.4)$ hours.

Using the $\chi^{2}$ test, the difference in the recovery rate between the two groups was not significant. This notwithstanding, the clinical results of this study clearly indicate that intravenous branched chain amino acids are at least as effective as lactulose in reversing hepatic coma.

Table 2 Clinical results in 34 patients included in study

\begin{tabular}{lll}
\hline & $\begin{array}{l}\text { Treatment A } \\
\text { (Branched chain } \\
\text { amino acid+ } \\
\text { glucose) }\end{array}$ & $\begin{array}{l}\text { Treatment B } \\
\text { (Lactulose+ } \\
\text { glucose) }\end{array}$ \\
\hline $\begin{array}{l}\text { No of patients } \\
\text { Responsive }\end{array}$ & 17 & 17 \\
Unresponsive & $12(70 \cdot 6 \%)$ & $8(47 \cdot 1 \%)$ \\
\hline
\end{tabular}

* Three patients died from bleeding; two patients received the combined treatment.

† Two patients died from bleeding; one from hepatorenal syndrome; one from acute liver necrosis; five patients received the combined treatment. 


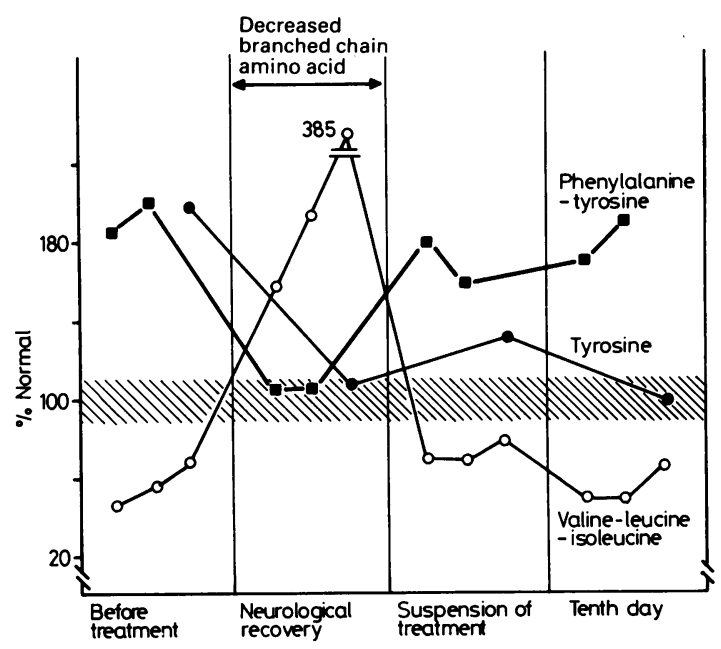

Fig. 5 Plasma aromatic amino acid and branched chain amino acid behaviour in patients with hepatic encephalopathy receiving intravenous branched chain amino acid.
In this study blood samples for determining amino acid were drawn before starting treatment, on mental recovery, six hours after the treatment was discontinued, and at the end of the study. Fig. 5 shows the behaviour of plasma aromatic amino acid and branched chain amino acid in the patients treated with branched chain amino acid. Phenylalanine, tyrosine, and tryptophan, which were high when patients were in coma, fell to normal limits on mental recovery. It was surprising and also puzzling to observe that, with the exception of free tryptophan, aromatic amino acid and branched chain amino acid had returned to pretreatment values at the tenth day, when patients had no evidence of encephalopathy.

The significant correlation between plasma free tryptophan concentrations and the mental state provides corroborative evidence for the role of raised tryptophan concentration in the pathogenesis of hepatic encephalopathy. It must also be appreciated, however, that changes in plasma concentrations do not necessarily reflect changes in brain concentrations. To investigate the false neuro-

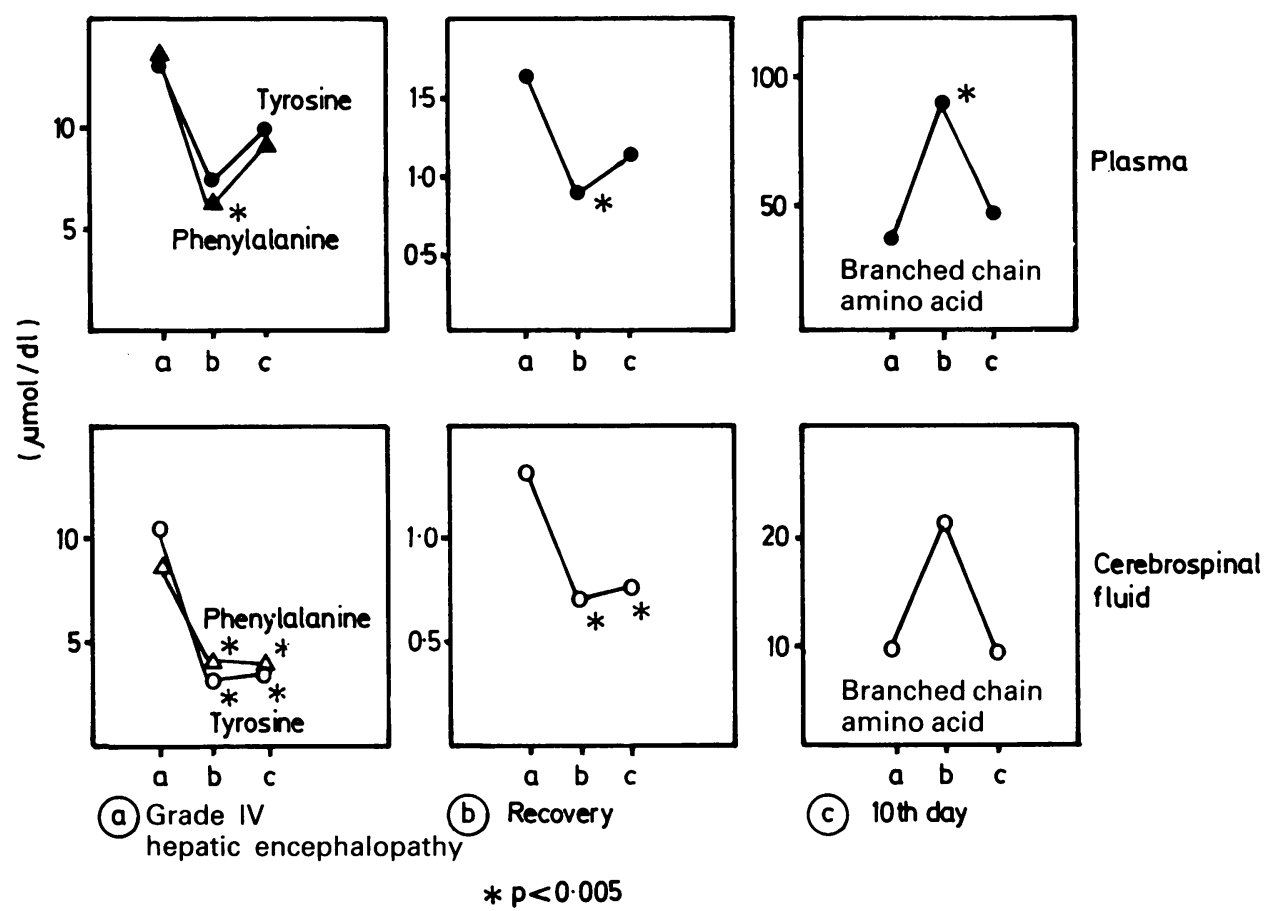

Fig. 6 Plasma and cerebrospinal fluid amino acid pattern in patients with hepatic encephalopathy receiving intravenous branched chain amino acid. 
transmitter hypothesis further it was decided to monitor simultaneously amino acid and biogenic amine concentrations both in plasma and cerebrospinal fluid. Fourteen patients with grade 3-4 hepatic encephalopathy received branched chain amino acid, using the same modalities as those in the previous study. Plasma and cerebrospinal fluid amino acid and amine concentrations were determined only in the 10 patients who regained consciousness. ${ }^{12}$ The amino acid pattern was found to be quite different in cerebrospinal fluid and plasma (Fig. 6). Cerebrospinal fluid concentrations of tyrosine and tryptophan had decreased considerably by the time the patients regained consciousness and remained within the normal range by the tenth day. By contrast, although plasma concentrations of tyrosine and tryptophan fell when consciousness was regained, 10 days later they had increased again (Fig. 6). These findings are mirrored by the finding that the concentrations of octopamine and phenylethanolamine in cerebrospinal fluid had also fallen on regaining of consciousness (Fig. 7). ${ }^{13}$

The data presented argue strongly in favour of a therapeutic effect of branched chain amino acid in the treatment of hepatic coma in patients with

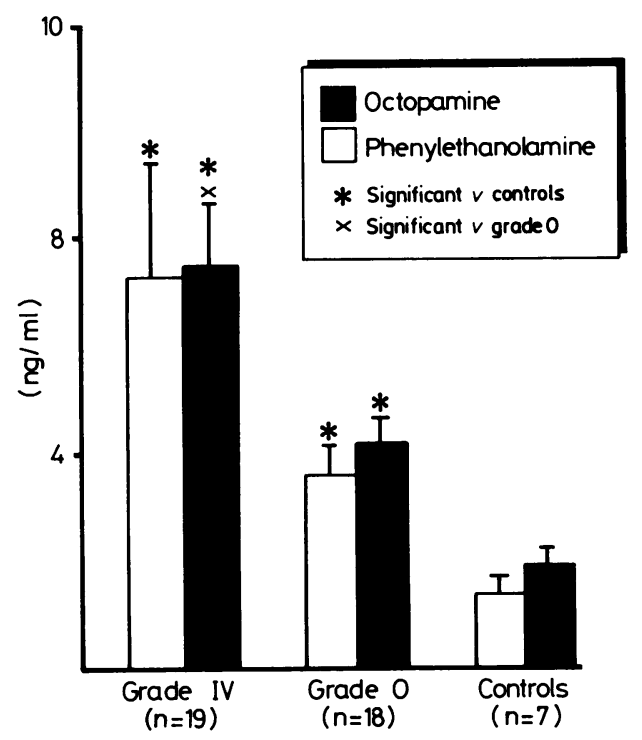

Fig. 7 Cerebrospinal fluid, octopamine, and phenylethanolamine concentrations in patients with hepatic encephalopathy receiving intravenous branched chain amino acid. chronic liver disease. Branched chain amino acids are likely to act via two distinct mechanisms; firstly, by inhibiting transport of aromatic amino acid across the blood brain barrier; and secondly, by reducing aromatic amino acid and ammonia outflow from muscles as a consequence of their regulatory effects on muscular protein turnover.

\section{References}

1 Cascino A, Cangiano C, Calcaterra V, Rossi Fanelli F, Capocaccia L. Plasma amino acid imbalance in patients with liver disease. Am J Dig Dis 1978; 23: 591-8.

2 Oldendorf WH, Szabo J. An assignment to one or three BBB amino acid carriers. Am J Physiol 1976; 230: 94-8.

3 Fischer JE, Baldessarini RJ. False neurotransmitters and hepatic failure. Lancet 1971; ii: 75-9.

4 Rossi Fanelli F, Freund H, Krause R, et al. Induction of coma in normal dogs by the infusion of aromatic amino acids and its prevention by the addition of branched-chain amino acids. Gastroenterology 1982; 83: $664-71$.

5 Scherwin RS. Effect of starvation on the turnover and metabolic response to leucine. J Clin Invest 1978; 21: 1471-81.

6 Freund HR, James JH, Fischer JE. Nitrogen sparing mechanisms of branched chain amino acids in the injured rat. Surgery 1980; 90: 237-43.

7 Sakamoto A, Moldawer LL, Bothe A, Bistrian BR, Blackburne GL. Are the nitrogen sparing mechanisms of branched chain administration really unique? Surgical forum 1980; 31: 99-100.

8 Rossi Fanelli F, Angelico M, Cangiano C, et al. Effect of glucose and/or branched-chain amino acid infusion on plasma amino acids imbalance in chronic liver failure. JPEN 1981; 5: 414-19.

9 Capocaccia L, Calcaterra V, Cangiano C, et al. Therapeutic effect of branched chain amino acids in hepatic encephalopathy: a preliminary study. In: Orloff MJ, Stipa S, Ziparo V, eds. Medical and surgical problems of portal hypertension. London: Academic Press, 1980: 239-49.

10 Rossi Fanelli F, Riggio O, Cangiano C, et al. Branchedchain acids vs lactulose in the treatment of hepatic coma. A controlled study. Dig Dis Sci 1982; 27: 929-35.

11 Adams RD, Foley SM. The neurological disorder associated with liver disease. Res Publ Assoc Res Nerv Ment Dis 1954; 32: 198-237.

12 Cangiano C, Cascïno A, Capocaccia L, et al. Modifications of plasma and CSF amino acid pattern in patients with severe hepatic encephalopathy receiving i.v. branched-chain amino acids. Journal of Clinical Nutrition and Gastroenterology 1986; 1: 133-8.

13 Riggio O, Fiaccadori F, Berto E, et al. Behaviour of cerebro spinal fluid false neurotransmitters in hepatic encephalopathy. A preliminary study. Ital J Gastroenterol 1985; 17: 78-80. 